\title{
Understanding Banking via WeChat Diaries
}

\author{
Jocelyn Sie", Wei Eng Koh", Shamil Zainuddin", Graham I. Johnson* \\ ${ }^{\#}$ Research, Ethnography, Design (RED), NCR, Singapore \\ E-mail: jocelyn.sie@ncr.com,weieng.koh@ncr.com, shamil.zainuddin@ncr.com \\ *Research, Ethnography, Design (RED), NCR, Dundee, Scotland \\ E-mail: graham.johnson@ncr.com
}

\begin{abstract}
This paper describes the development and refinement of an existing chat application, China's WeChat, as a remote diary tool, which researchers can employ to acquire a deeper understanding on their consumers whilst overcoming major geographical and time zone constraints. This research was part of a project to understand the experiences of Chinese consumers related to finance and banking. A diary study was conducted through WeChat and phone interviews to understand how consumers conduct their everyday financial transactions. The research illustrated the need to adapt approaches to diary participants, such as utilising a chat agent or avatar to elicit richer data and demonstrated the utility of a feedback loop, which reassured and reminded participants to post regularly, in turn encouraging posts with more depth. The relationships built over the course of the diary study were also imperative to the success of follow-up semi-structured phone interviews, as trust and familiarity between researchers and participants enabled more intimate conversations. Novel use of the application worked well in gaining a deeper appreciation of the experiences of selected consumers. The paper adds to the growing literature on the use and adaptation of chat applications as a substitute for conventional mobile diary tools and concludes with a list of key considerations for further applications in a similar research context.
\end{abstract}

Keywords - remote research; methodology; ethnography; diary studies; avatar; mobile HCI; consumer experience; visual agent

\section{INTRODUCTION}

Technology companies whose solutions such as ATMs or payment kiosks are used globally by millions daily, need to gain an understanding of consumers' requirements, preferences and lived experiences to inform strategy, marketing, and development. Behaviour and consumption habits can differ greatly based on culture, media influences, family and peers, attitudes, income, and demographics. However, exploring people's daily lives and uncovering specific user requirements can be challenging, especially when users are spread across the globe.

Quantitative research adopting surveys can have a wide reach through digital media and can provide general results, which may raise further questions [1]. Survey results can often miss underlying reasons, real-world behaviour, and core motivations behind consumer choices, something that well-designed qualitative research aims for. As the need for deeper consumer insights grows, surveys are increasingly complemented with diaries, where participants record their relevant activities and reflections over a certain period [2], [3].

This research explored the use of digital qualitative research tools that enable the capture of in situ data (e.g. actions, feelings, thoughts) of consumers remotely, to overcome significant challenges with time, scale and cost very often associated with qualitative research. This paper describes key lessons learned from adapting a popular and sophisticated chat app, WeChat [4], as a remote qualitative data collection tool, illustrating the challenges, refinement, and results of this approach.

\section{A. Related Works}

\section{1) Mobile Diary Benefits}

One approach to understanding consumers is via immersion into consumers' lives and culture, observing and shadowing users in situ. However, time, geographical constraints, intrusion, and privacy are factors to be considered. A potentially less intrusive approach is via diaries, where participants document what they do in a specific setting, or when engaging with a particular product, task, or service. Mobile diaries, which can be attempted on the go, are ideal for longitudinal studies because they can record patterns of behaviour and provide a wealth of detailed information about participants' habits and motivations. The chief advantage of using the mobile diary to access personal financial behavior and thoughts was in being able to record in situ, at a distance, at no real cost, and easily, on a regularly used personal device that can capture images/video. 
Diary study has its roots in both psychological and anthropological research while being widely applied in social science, psychology, and HCI [5], [6]. Although data collected is subject to participants' willingness to share, such data can assist prior to on-site visits or interviews, and in establishing the authenticity of the information provided by participants [7].

Mobile diaries can be conducted in many ways, such as providing participants with disposable cameras, notebooks or audio recorders to log their day-to-day activities [8]. Using a mobile device as a medium reduces the need to educate participants already owning smartphones and enables them to report their activities promptly, eliminating a time window that might cause participants to forget or dilute details of activities [6], [9], [10]. This approach can be an advantage over traditional interviews, which can rely on participant's memory. With an interactive diary, the ability to instantaneously respond to participants also improves rapport and a sense of co-presence, without the pressure of face-to-face contact [11].

\section{2) Mobile Diary Benefits}

While a diary can provide the voice of the consumer, the method is not without its drawbacks. One concern is the ability for researchers to motivate participants, with little to no face-to-face interaction, possibly resulting in poor understanding of problems or the reality of how the research is conducted on the participant's end [12]. Another challenge is the reliability of self-reported data where participants may have a strong sense of self-awareness [13] or response bias [14] and self-censor stories that might not accurately reflect the actual activities. Though this heightened sensitivity can also be looked upon as a reflection of participants' aspirations [12].

The longitudinal nature of diaries commonly causes participant fatigue and exhaustion, resulting in a drop in both the amount and quality of recorded data [15]. As the purpose of the mobile diary method is to acquire an in-depth understanding, the importance of interacting with participants in a more personalised manner is important. Participants might not always carefully follow instructions, so a communication channel for participant feedback is required [16].

\section{3) Chat Agents and Avatars}

Virtual characters that can communicate range from nonanimated simple avatars to Embodied Conversational Agents (ECA) [17], [18]. The former can vary in expressions and latter can use artificial intelligence to provide a degree of humanness, both of which can increase persuasiveness and socialness [19]. This paper also addressed a non-animated avatar, created during the course of our mobile diary study in an effort to increase or maintain participants' motivation to continually participate and the encouragement to share details via the diary.

\section{MATERIAL AND METHOD}

The mobile diary approach employed gave researchers in Singapore the opportunity to remotely gather information on consumers' lifestyles and financial behavior, before investing resources in field studies within China.
Participants completed diaries and partook in semistructured phone interviews, in which they reported on the types and context of financial transactions they had conducted within the duration of the study, identified their common transactions and preferred channels for their transaction needs.

The choice to conduct diary studies via smartphones was mainly due to the high penetration of 1.289 billion smartphones users in China [20]. Existing chat apps were considered to enable researchers to simultaneously interact with several users, and participants could avoid purchasing and experiencing potential difficulties using a new app or learning a new documentation method [12], [21]. The intent was to leverage participants' existing use of such messaging systems to communicate their lives with friends and family [10]. In a similar style, we wanted participants to share their daily transactional lifestyle via their personal mobile devices, capturing (via text, photos, audio) activities on the go that might be lost during interviews or not easily observed in the field.

The longitudinal aspect of this approach was deliberate. It was not uncommon for participants to perform main banking transactions only once or twice a month.

\section{1) Chat Application Alternatives Considered}

A suitable and cost-free application to host remote diaries was imperative to the success of the research. As the study involved recruitment of participants from China, popular chat apps in Asia like Google Talk, Facebook's Messenger and WhatsApp, Korea's KakaoTalk or Japan's Line Messenger were disqualified, considering China's strict data restrictions [22]. Instead, China-based social media and messaging apps were shortlisted, namely Weibo, WeChat, and QQ. These tools provided an array of options, not only messaging services (traditional and app-based chat services), but also tools that allow users to post updates to their "friends' circle" on their accounts.

An evaluation of each platform was conducted by creating accounts, testing the usability, privacy, and ease to export data out for compilation and analysis. The main rationale for choosing WeChat was its growing and widespread popularity, with reportedly 750 million users [23], allowing us to take advantage of participants' familiarity with it. Although the name of the app suggests chat services, it also has a social media feature, allowing users to share posts with selected friends on their individual walls. Gaining access to users' social media walls after participants added the project account as a "friend" provided researchers a deeper view of the lifestyle and behaviour of participants, allowing for cross-referencing wall postings with data collected from their diaries.

Similarly, participants who added researchers as friends were able to find information on the objective of the study and download related content from the researcher's wall (Fig. 1). 


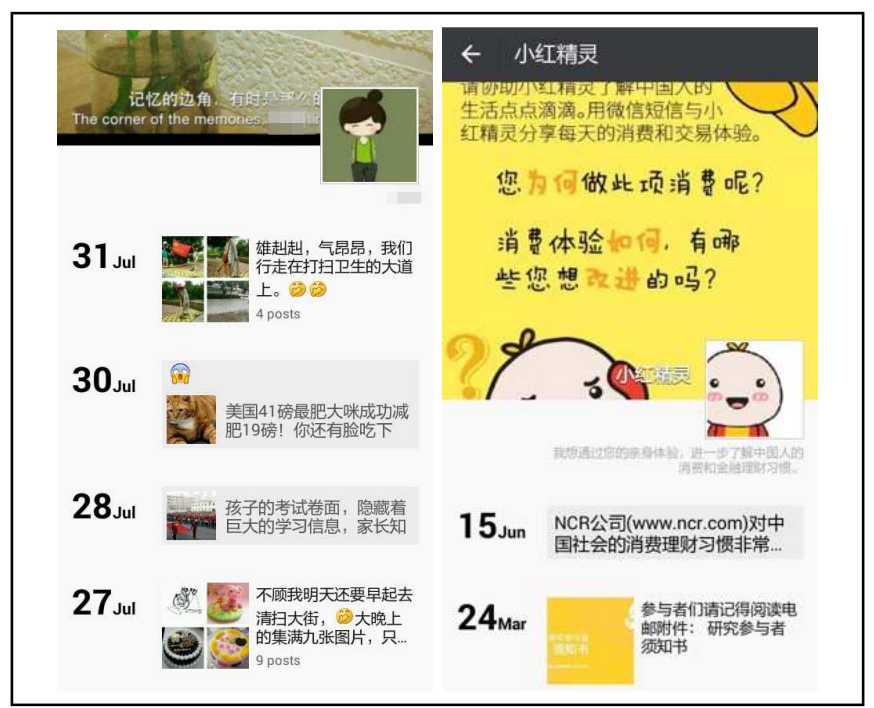

Fig. 1 Participants' WeChat wall sharing photos and articles, similar to a Facebook Wall (Left). Researcher's WeChat profile stating details and focus of the diary study (Right)

\section{2) Participants}

We conducted five studies through WeChat in Mandarin with 40 Chinese participants in total, continuously exploring ways to encourage detailed postings of personal transactions from participants, iterating and refining our approach with each subsequent study. The study lengths varied from 7 days for a pilot, to 30 days. All participants owned smartphones, in a mix of local brands (Lenovo, Huawei) and international brands (Samsung, Apple), and were all current users of WeChat.

For the first two studies, we used convenience sampling where personal contacts were recruited to help engender trust between researchers and participants, given the sensitivity of the research topic. Then, participants were asked for recommended contacts at the end of those. This method of snowball sampling worked well and was repeated for the next two studies. All four studies involved participants from ages 25-35, with a mix of students and white-collared workers. To widen participant diversity, the last study made use of a recruitment agency for 20 participants from ages 25-57, living in various parts of China from Tier 1 cities such as Tianjin and Shanghai to Tier 3 provinces such as Shandong and Liaoning.

\section{3) Ethical Concerns}

The distance between researchers and participants meant this research needed more effort to make sure ethical concerns were addressed [11]. Participants were informed of the study's requirements through phone calls and emails, highlighting their right to privacy and confidentiality, and how their data would be handled. Formal introductions and a QR code linking to researchers' WeChat profile were then emailed upon written consent to the study.

Given the length and personal nature of the research, we were mindful how the relationship established with participants depended upon trust, especially given the release of personal information. Studies 2 and 3 received photos revealing bank account balances and investment details.
It was important for expectations and goals between participants and researcher to be constantly negotiated via reminders of the research objective and explaining how the information was used [24]. Thus care was taken to emphasise participants' confidentiality and their freedom to edit photos (Fig. 2) containing sensitive information such as bank details.

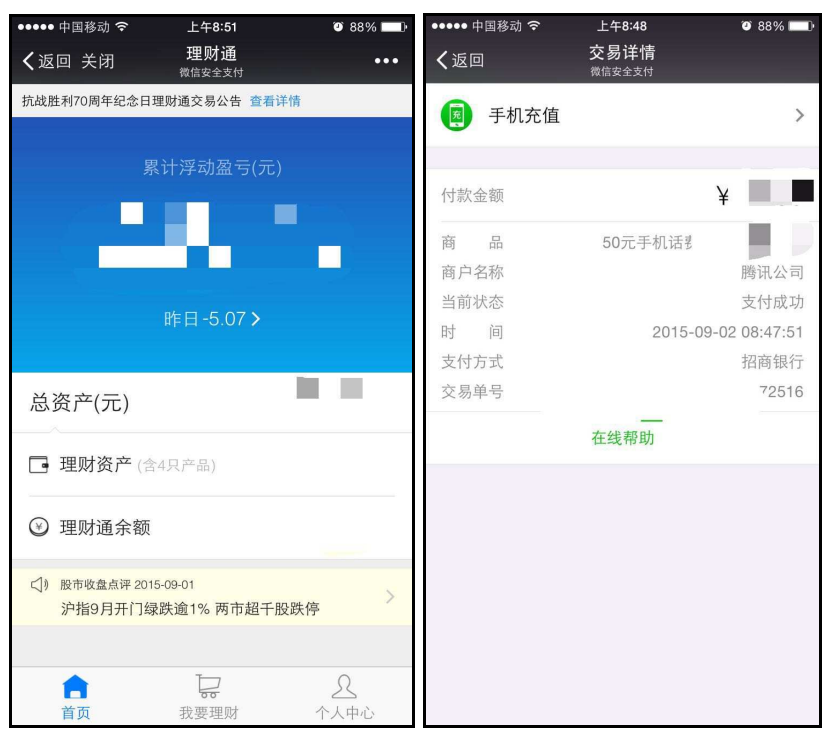

Fig. 2 Edited screenshots posted by participants

\section{4) Method}

Participants were asked to post via WeChat, in a private chat room with researchers, their daily expenses, environment and reflections on their transactions in a combination of photos, videos, audio, or text messages, before, during, or after a financial activity, which may include paying for meals, withdrawing money or visiting a banking website. Asking for images adds a depth not normally possible through interviews. Instead of requesting details and descriptions of every transaction as we did in Study 1, instructions for the next studies shifted to getting participants to provide us with the background (e.g. why was the said transaction made) and environment where transactions had taken place.

Initial attempts with descriptive texts and example posts proved insufficient as an explanation. Participants reported that they did not realise heading to an ATM was considered a transaction, nor was treating friends to a meal. Hence scenarios and examples were highlighted and in subsequent studies drawn out graphically (Fig. 3). 


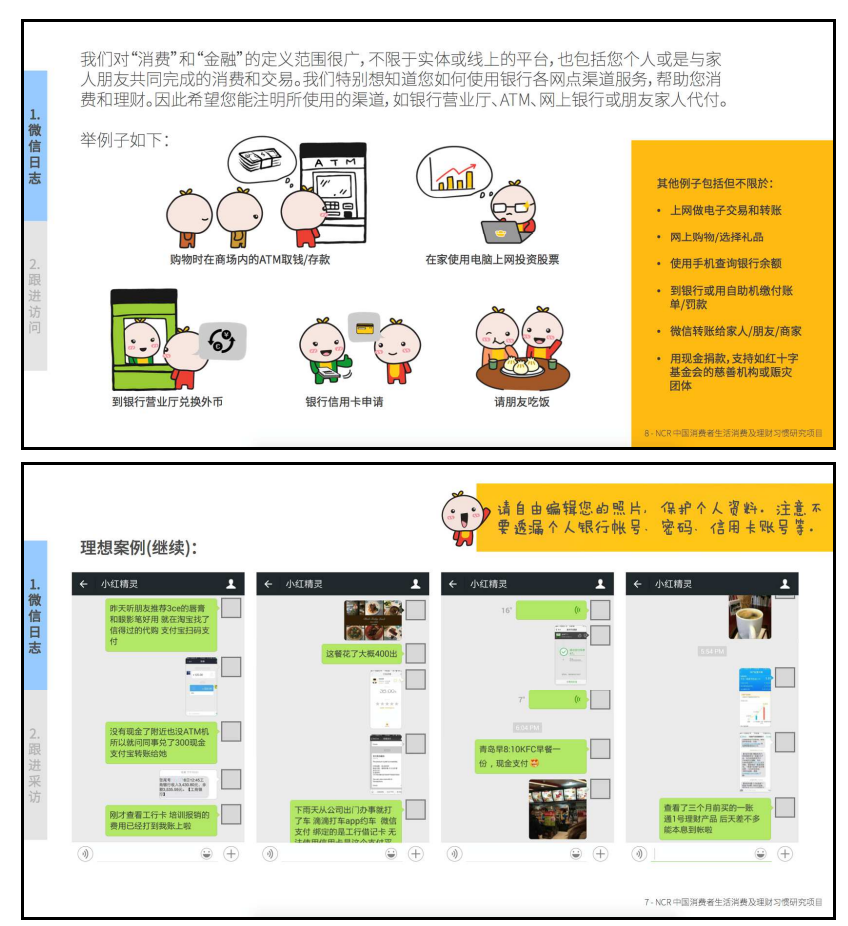

Fig. 3 Instructions sent to participants, illustrating examples of post preferred during the course of the study like gifting money to a friend or checking account details online

\section{RESULTS AND DISCUSSION}

Lessons from our previous rounds of studies were used to better the design of this novel diary approach.

\section{1) Visual Agent}

Reflections from Studies 1 and 2 noted participants being open with their thoughts and reflections due to the existing relationship with the researcher. However, as subsequent studies involved participants with no initial connection to the researcher, participants seemed less willing to provide their reflections.

We noticed participants' posts by-passed cultural explanations, as they tended to assume the diary facilitator understood all the nuances behind the activity. For example, participants posted "I spent $\$ X$ on taxi today" but stopped short of explaining how exactly the payment was made. It was only via follow-up interviews that we found that taxis are commonly hired and paid via mobile platforms in China. Also, participants seemed to equate the diary to a financial tracking app and did not feel it necessary to further explain their choices.

Naturally, it might feel invasive to know that intimate data-what they saw, where they were, how they felt-was collected and analysed by people they have never met. The aim was to increase quality and quantity of diary posts to inform formulating of in-depth phone interview questions. It became apparent that for the approach to work well there was a need to have a means of encouraging, prompting and reminding with regard to diary entries.

An additional challenge was that many were less open in their posts and protective about personal finances. Participants' trust had to be gained and a relationship established with the research team within the WeChat environment, which is both social and conversational in nature. It was with these challenges in mind that initial thoughts turned to an agent that acts as a means of improving both quantity and quality of dialogue with distant and varied participants. Such a character or agent needed to be friendly, approachable, culturally-appropriate, and representative of a curious outsider.

Acknowledging previous work on avatars and cultural probes [16], [25], [26] the team began considering options for a neutral, functional character that met the requirements outlined above. The visual agent was introduced as a curious cartoonish alien named Little Red Sprite wanting to understand 'Earthlings', to incite participants to document in more detail, explaining their activities and all relevant Chinese cultural aspects - just as they would do so for a non-local, instead of assuming researchers have a background understanding of China. (Fig. 4).

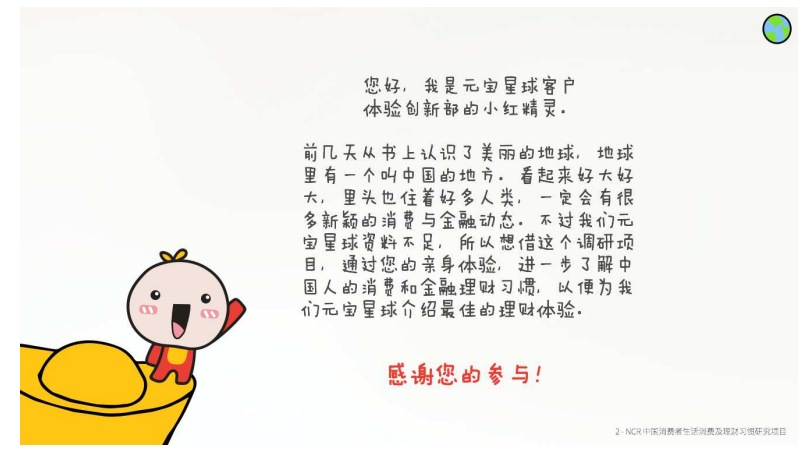

Fig. 4 The rationale behind designing 'Little Red Sprite' as a nonhuman was to encourage participants to explain their common daily occurrences and transactions as they would to foreigners outside China

Early feedback from the pilots noted that researchers were communicating too formally, which did not assist the overall goals. Thus, along with designing the agent to look more endearing, we took a leaf from the mascots of China's Internet giants [27], [28] (Fig. 5) and changed the tone to be more colloquial and playful.
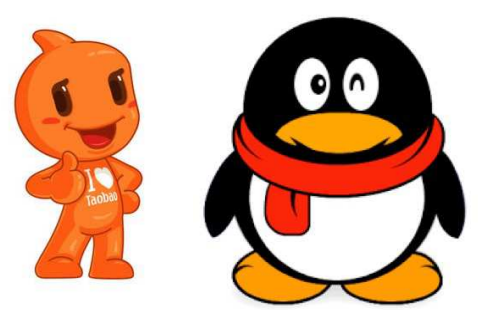

Fig. 5 Alibaba's Taobao (Online shopping site) Mascot淘公仔 Tao Doll (Left) and Tencent's QQ (Mobile messaging platform) Mascot QQ 企鵝 QQ Penguin (Right)

Wanting to be more personable to elicit higher quality and quantity of posts [16], we leveraged WeChat's sticker culture to engage in dialogue and reply to posts with customized stickers (Fig. 6). The role of Little Red Sprite was critical here to prevent participants from being intimidated or overwhelmed by the requirements of the research. We believed an advantage of this type of avatar was in its social capability, essential to the continued dialogue. Care was taken to have Little Red Sprite display 
emotions of gratitude and curiosity, as opposed to disappointment, say when posts had not been made.

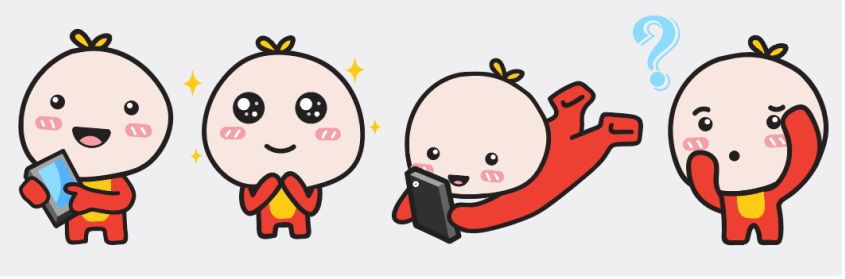

Fig. 6 "Little Red Sprite" as customised WeChat stickers for conversations with participants

The agent seemed to resonate well with all participants and responses were open and friendlier, especially during the follow-up interviews where participants addressed the researchers as 'Little Red Sprite'. Participants also apologized through WeChat for not spending money or not posting - these were responses we had never received from previous (non-agent) studies. Posts also had more explanations and included participants' comments like "Oh yes, you might not know, but in China we ...".

\section{2) Reminders and Encouragement}

One of the biggest challenges participants reported was the difficulty in remembering to capture the moment, as it happened. Another was remembering to post their transactions. Participants described how they were in the midst of posting when their smartphone rang or they had to attend to other matters. To avoid simplistic posts, constant reminders that posts did not always have to be about the price of expenditure and to include reflections were sent to participants. Examples of demand characteristics observed were posts by participants requesting affirmation, posts of participants apologizing for not spending money, and posts of receipts only.

Feedback from Study 1 noted reminders were annoying to receive first thing in the morning and it reflected a lack of trust that participants would document their transactions. Thus, in Study 2, customized countdown (Fig. 7) acting as reminders and progress reflection were sent nightly but participants mentioned feeling guilt-tripped on seeing that their lacks of posts were recorded. With each study, efforts to remind and encourage grew. Follow-up interviews showed us how participants were desensitised to the nightly reminders after a week or so, thus there was a need to make sure the reminders changed visually. The following studies varied both colours and messages to encourage participants. By Study 5, instead of countdowns, our nightly posts included tips on how to record their posts.

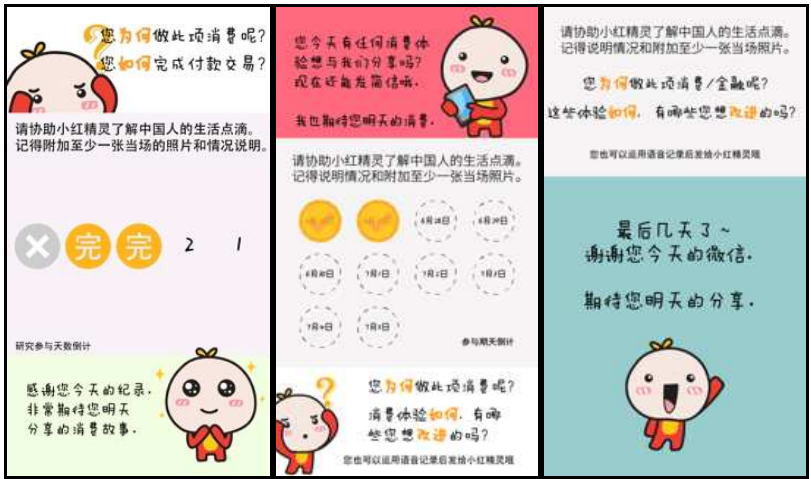

Fig. 7 Countdown indicators evolved from reflecting response; grey $\mathrm{X}$ for a day with no posts, orange circle with the Chinese character for "Finish" for a day with posts (Left), to noting exact dates within each circle and sending an orange tick for every day passed (Middle), to no countdowns but daily statements letting them know; their posts had been read, their posts were interesting, it was ok to backdate posts and so on (Right)

Largely, reminders were received positively with participants saying it helped and it was more interesting than finance-tracking apps, which are usually passive. Across the studies, it was common to have participants continuing to post their transactions and reflections even after they have received notice that the study had ended.

\section{3) Supplementary Questions}

Given flexibility to post about any transaction, some participants tended not to post common transactions such as topping up their transport card. Reasons for not posting such transactions from the follow-up interviews; "It was so common I didn't think I need to post about it" and "I do it so often I actually forget it's a transaction". Thus participants in Study 4 were sent questions about specific transactions after we noticed nothing about them have been posted (Fig. 8).

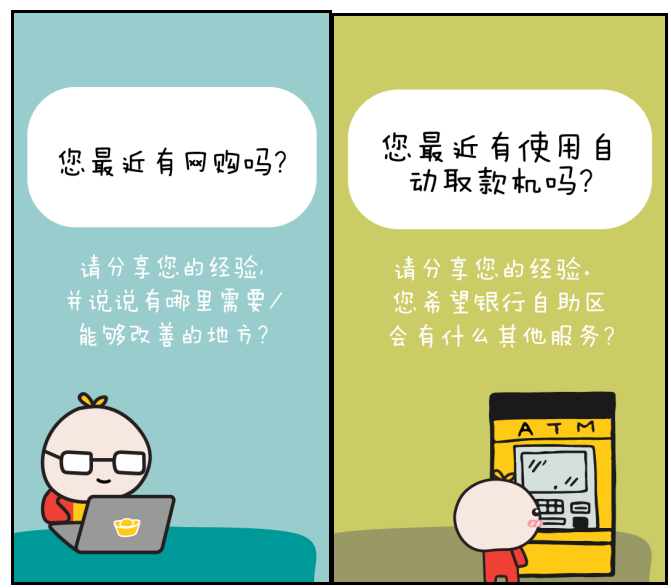

Fig. 8 Questions were sent to participants periodically if they had yet to post about common transactions such as: "Have you done online shopping recently?", "Have you visited the ATM recently?" and "Have you done Internet banking recently?" 


\section{4) Dialogue Through WeChat}

With the month-long studies, participants appeared unacknowledged, given that the only responses were countdown calendars or one-sided questions. With time, posts started to be simpler, less frequent and questions like "Am I doing this right?" were appearing. It was crucial to close the loop while verifying our interpretations. Participants were questioned directly on WeChat posts; such as "You mentioned heading to the supermarket with your credit card. Were there specific benefits of using a credit card?" or "I noticed your posts hardly mention about shopping online. Do you usually shop online?"

Dialogues not only demonstrated the kind of reciprocity that was inherently motivating but also helped us in drawing a detailed picture of activity. The WeChat conversations also helped scope questions for the follow-up interview phase, allowing for more in-depth questions like "Could you explain why you have specific credit cards for shopping online and shopping offline?"

\section{5) Incentives}

For the first three studies, no incentives were provided but through follow-up interviews with participants, many expressed that the idea of getting feedback or a glimpse of what they have helped contribute to was appealing. That was acted on in Study 4 where participants were incentivised with an understanding report of their posted transactions. Participants expressed how receiving a personalised report (Fig. 9) of their spending habits was very helpful. But due to the additional effort required to produce such reports, the decision was made to incentivise participants with RMB500 each through a recruitment agent in the Study 5. Though, at the end of the study, we accepted requests from participants who wanted a summary of their input.

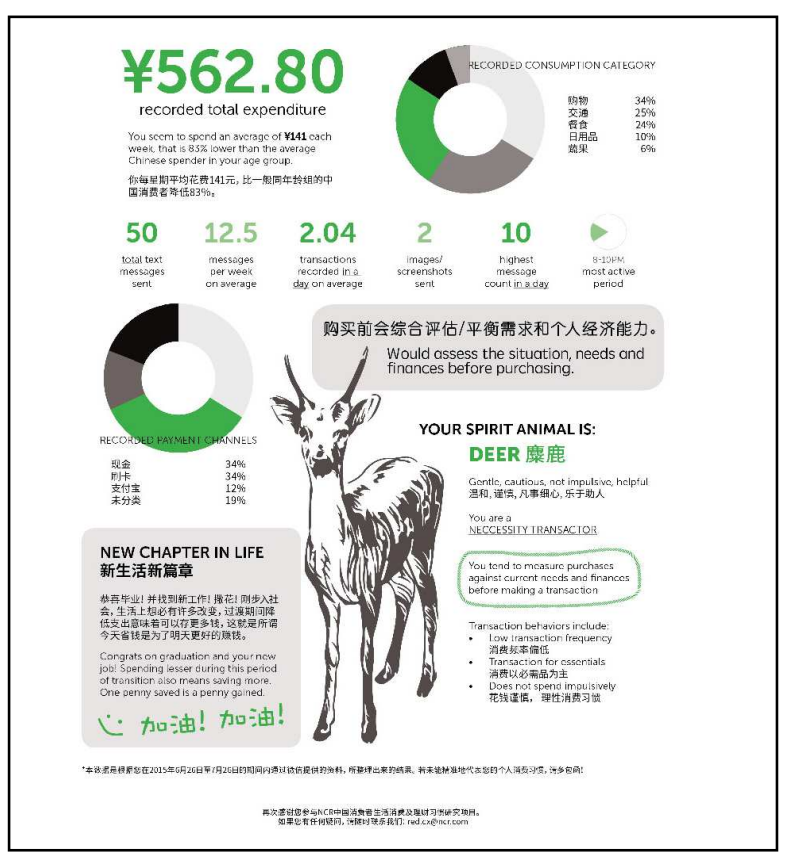

Fig. 9 Study 4 participants received a personalised report as incentive

\section{6) Follow-up Interviews}

Conducting phone interviews helped identify the obstacles experienced by participants, limitations in the tool, as well as supplementing the data collected from the diary. This was especially true for sensitive or complicated questions for WeChat such as "Why did you buy this investment product now and who did you consult with prior to the purchase?"

Also, participants were more responsive during interviews, compared to on WeChat. Reasons like lack of feedback from researcher via WeChat or the belief that they were interacting with a machine and not a person were given to explain differences in enthusiasm. Also, many noted exercising caution about their mobile data usage and hence limiting details in their posts or only posting photos when they were home.

While phone interviews presented some challenges to data collection, such as the absence of body language and cues, they were not a significant barrier to the collection of quality data. The main challenges instead were coordination, timing, and language. As participants varied from managers to housewives to retirees, their available timings were sporadic; calls were scheduled either late at night or early mornings, and often interviews were interrupted by incoming calls, a baby's cry, or sudden house visits.

Though both researchers and participants were fluent in Mandarin, the speech varied between participants, based on where they came from as well as their age. Older participants would speak with stronger accents similar to their native dialects, making it hard for researchers to discern their speech. For younger participants, terms they used were not always straightforward. For example, “AA" refers to 'going Dutch' while the Moonlight Crowd (月光族) are people who squander their pay check every month. Fortunately, follow-up interviews allowed researchers to clarify such terms easily.

\section{IV.CONCLUSION}

This research clearly demonstrated the success of an adapted mainstream messaging app as a remote research tool, a viable diary approach to consumer experience investigation. The overall success of the avatar technique was measured by the higher quality data (amount of detailed information) provided by participants, and by increased engagement (how frequently participants posted). For some, posting even continued after receiving notice that the study had ended. Another telling factor was that the relationships developed (with Little Red Sprite) led to the success of the follow-up phone interviews, as trust and familiarity between researchers and participants enabled more open, informal and detailed conversations.

Our results from the studies supplemented field research done in China and enabled for mapping of a typical Chinese banking journey, highlighting associated challenges and opportunities for innovation.

Compared to other mobile research tools such as dscout [29] or Validately [30], an existing chat app not only reduces the time needed for participants to learn, the nature of a chat app also encourages conversations, as participants are 
familiar with such applications for constant and consistent communication.

The demand on the researcher-participant interaction of such an approach tool is for openness, responsiveness, and follow-up. Being able to view participants' social wall provided more context into the lives of participants without researchers having to pose too many questions.

\section{1) Challenges}

The iterations carried out between the mobile diary studies were a function of the learning gained, with challenges met in a number of innovative ways.

For the first few studies, participants' not attending to the instructional materials sent was noted. Participants were asking questions about what was required, what the project was for and when do they start. This highlighted the importance for taking participants through instruction materials after recruitment instead of merely handing them materials to look through.

Another challenge we overcame was establishing trust with participants. In recruitment attempts, some were apprehensive upon realising researchers were based in Singapore, or from a non-local company. Because the study required a connection with them via $\mathrm{WeChat}$, which to many is a personal tool they use to connect with friends and family, such reactions were expected. Establishing an understanding of how their responses will be of value was a stronger reason for them to contribute more expressively and regularly.

The major challenge in all the diary studies was maintaining a high level of participant commitment to ensure both quality and quantity of data. It was difficult to manage participants over a long period of time, where there was a sense of repetition and responses became more superficial and less detailed. The longer the study time, the more feedback was needed to keep participants active.

Internet access was also a factor when it came to considering the feasibility of using instant messaging apps for diary studies as not all participants had, or chose to have, constant Internet connection. To manage their data consumption, some participants mentioned only sending their collated posts when they have access to Wi-Fi in their offices or at home, instead of paying for data usage on the go.

Engaging participants to continuously post about their day required vigilance and creativity in feedback and encouragement. However, the data gathered from the longer studies were much richer and with the follow-up phone interviews we were able to gain an in-depth understanding.

Also, the creation of this avatar, from scratch, required a degree of creativity and design skills, something future researchers interested in this technique should note.

\section{2) Suggestions for Future Research}

Depending on cultural differences and market preferences, we may find differing usage patterns of the mobile chat in other countries. Chat apps popular in other markets, e.g. LINE and WhatsApp are designed with unique features and a research approach could be varied to engage participants differently. Future research is therefore required to explore further the viability of the chat app adaptation as a remote consumer research tool in different places and cultural contexts.

Also, when adopting the avatar approach we must be cognizant of the importance of cultural and 'audience' factors, which in retrospect were key to the success of Little Red Sprite.

\section{ACKNOWLEDGMENT}

The authors' gratitude goes to all participants, and to NCR Corp. for support of this research.

\section{REFERENCES}

[1] J. Ilieva, S. Baron and N. Healey, On-line surveys in international marketing research. Manchester: Graduate School of Business, Manchester Metropolitan University, 2001.

[2] Bagnall, D. Bounie, K. Huynh, A. Kosse, T. Schmidt, S. Schuh and H. Stix, "Consumer Cash Usage: A Cross-Country Comparison with Payment Diary Survey Data", SSRN Electronic Journal.

[3] N. Jonker, A. Kosse and L. Hernández, Cash usage in the Netherlands: how much, where, when, who and whenever one wants? Amsterdam: De Nederlandsche Bank, 2012.

[4] "WeChat - Free messaging and calling app", Wechat.com, 2016. [Online]. Available: http://www.wechat.com/en/

[5] R. Gillham, "Diary Studies as a Tool for Efficient Cross-Cultural Design", in 7th International Workshop on Internationalisation of Products and Systems, Amsterdam, The Netherlands., 2005.

[6] X. Sun, D. Golightly, J. Cranwell, B. Bedwell and S. Sharples, "Participant Experiences of Mobile Device-Based Diary Studies", International Journal of Mobile Human Computer Interaction, vol. 5, no. 2, pp. 62-83, 2013.

[7] S. Carter and J. Mankoff, "When participants do the capturing", Proceedings of the SIGCHI conference on Human factors in computing systems - CHI '05, 2005.

[8] W. Gaver, A. Boucher, S. Pennington and B. Walker, "Cultural probes and the value of uncertainty", interactions, vol. 11, no. 5, p. 53, 2004.

[9] J. Brandt, N. Weiss and S. Klemmer, "txt 4 18r", CHI '07 extended abstracts on Human factors in computing systems - CHI '07, 2007.

[10] K. O'Hara, M. Massimi, R. Harper, S. Rubens and J. Morris, "Everyday dwelling with WhatsApp", Proceedings of the 17th ACM conference on Computer supported cooperative work \& social computing - CSCW'14, 2014.

[11] C. Tagg, A. Lyons, R. Hu and F. Rock, "The Ethics of Digital Ethnography in a Team Project. Working Papers in Translanguaging and Translation (WP. 12)".

[12] S. Hulkko, T. Mattelmaki, K. Virtanen and T. Keinonen, "Mobile probes", Proceedings of the third Nordic conference on Humancomputer interaction - NordiCHI '04, 2004.

[13] E. Appleton, "Market Research and Mobile Self-Ethnography - An Interview With Siamack Salari", GreenBook, 2014. [Online]. Available: http://www.greenbookblog.org/2014/03/18/marketresearch-and-mobile-self-ethnography-an-interview-with-siamacksalari/

[14] M. Orne, "On the social psychology of the psychological experiment: With particular reference to demand characteristics and their implications.", Prevention \& Treatment, vol. 5, no. 1, 2002.

[15] T. Schmidt, "Consumers' Recording Behaviour in Payment Diaries Empirical Evidence from Germany", Survey Methods: Insights from the Field (SMIF), 2014.

[16] B. Gaver, T. Dunne and E. Pacenti, "Design: Cultural probes", interactions, vol. 6, no. 1, pp. 21-29, 1999.

[17] Q.V. Liao, M Davis, W. Geyer, M. Muller, and N.S. Shami, 2016, June. What Can You Do?: Studying Social-Agent Orientation and Agent Proactive Interactions with an Agent for Employees. In Proceedings of the 2016 ACM Conference on Designing Interactive Systems (pp. 264-275). ACM.

[18] J. Cassell, J. Sullivan, S. Prevost and E. Churchill. 2000. Embodied conversational agents. MIT Press, Cambridge, MA.

[19] J. Hyde, E.J. Carter, S. Kiesler, and J.K. Hodgins. 2015, April. Using an interactive avatar's facial expressiveness to increase persuasiveness and socialness. In Proceedings of the 33rd Annual ACM Conference on Human Factors in Computing Systems (pp. 1719-1728). ACM.

[20] "China: mobile users by month 2016 | Statistic", Statista, 2016. [Online]. Available: http://www.statista.com/statistics/278204/chinamobile-users-by-month 
[21] A. Patterson, "Processes, relationships, settings, products and consumers: the case for qualitative diary research", Qualitative Mrkt Res: An Int J, vol. 8, no. 2, pp. 142-156, 2005.

[22] S. Lee, "China tells South Korea it blocked KakaoTalk, Line to fight terrorism", Reuters, 2014 [Online]. http://www.reuters.com/article/2014/08/07/us-southkorea-china-appsidUSKBN0G709E20140807.

[23] C. Hatton, "Is Weibo on the way out?", BBC - China Blog, 2015.

[24] A. Markham and E. Buchanan, "Ethical considerations in digital research contexts", in Encyclopedia for Social \& Behavioral Sciences, 2nd ed., J. Wright, Ed. Elsevier Press, 2015, pp. 606-613.

[25] M. Scott, L. Pereira, and I. Oakley. 2014. Show me or tell me: Designing avatars for feedback. Interacting with Computers, iwu008.
[26] A.L. Baylor. 2009. Promoting motivation with virtual agents and avatars: role of visual presence and appearance. Philosophical Transactions of the Royal Society of London B: Biological Sciences, 364(1535), 3559- 3565 .

[27] "Taobao" - Online shopping, 2016. [Online]. Available: https://world.taobao.com/

[28] "QQ" - Mobile application software, 2016. [Online]. Available: http://im.qq.com/

[29] "dscout - Mobile moments-based research", dscout, 2016. [Online]. Available: https://dscout.com

[30] "Validately - Test a feature before you write code", Validately.com, 2016. [Online]. Available: https://validately.com/ 